\title{
THE LUMPED MASS FINITE ELEMENT METHOD FOR A PARABOLIC PROBLEM
}

\author{
C. M. CHEN ${ }^{1}$ AND V. THOMÉE ${ }^{2}$
}

(Received 15 February 1983; revised 20 March 1984)

\begin{abstract}
For the heat equation in two space dimensions we consider semidiscrete and totally discrete variants of the lumped mass modification of the standard Galerkin method, using piecewise linear approximating functions, and demonstrate error estimates of optimal order in $L_{2}$ and of almost optimal order in $L_{\infty}$.
\end{abstract}

\section{Introduction}

Let $\Omega$ be a convex plane domain with smooth boundary $\partial \Omega$, and consider the initial boundary value problem

$$
\begin{array}{ll}
u_{t}-\Delta u=f & \text { in } \Omega \times[0, \infty), \\
u=0 & \text { on } \partial \Omega \times[0, \infty), \\
u(\cdot, 0)=v & \text { in } \Omega,
\end{array}
$$

where $u$, denotes $\partial u / \partial t$ and $\Delta$ the Laplacian $\partial^{2} u / \partial x_{1}^{2}+\partial^{2} u / \partial x_{2}^{2}$.

For $h$ small, let $\mathscr{T}_{h}$ be a partition of $\Omega$ into disjoint triangles $\tau$ with side lengths at most $h$, such that no vertex of any triangle lies on the interior of a side of another triangle, such that the angles of the triangles are bounded below independently of $h$ and such that the union of the triangles determine a polygonal domain $\Omega_{h}$ whose boundary vertices lie on $\partial \Omega$. Let then $S_{h} \subset H_{0}^{1}(\Omega)$ be the standard finite element space of continuous functions in $\Omega$ which reduce to linear functions on the triangles of $\mathscr{T}_{h}$ and vanish outside $\Omega_{h}$. With $\left\{P_{j}\right\}_{1}^{N_{h}}$ the interior vertices of $\mathscr{T}_{h}$ we shall employ the standard basis $\left\{\varphi_{J}\right\}_{1}^{N_{h}}$ for $S_{h}$ consisting of the pyramid functions defined by $\varphi_{j}\left(P_{k}\right)=\delta_{j k}$.

\footnotetext{
${ }^{1}$ Department of Mathematics, Xiangtan University, Xiangtan, Hunan, People's Republic of China.

${ }^{2}$ Department of Mathematics, Chalmers University of Technology, S-412 96 Göteborg, Sweden.

(c) Copyright Australian Mathematical Society 1985, Serial-fee code 0334-2700/85
} 
Recall that the standard semidiscrete Galerkin method for (1.1) is to find $u_{h}$ : $[0, \infty) \rightarrow S_{h}$ such that with $(\cdot, \cdot)$ the appropriate $L_{2}$ inner products,

$$
\begin{aligned}
\left(u_{h, t}, \chi\right)+\left(\nabla u_{h}, \nabla \chi\right) & =(f, \chi) \quad \forall \chi \in S_{h}, \text { for } t \geqslant 0, \\
u_{h}(0) & =v_{h},
\end{aligned}
$$

where $v_{h}$ is some approximation of $v$ in $S_{h}$. Recall also that this method may be written in matrix form as

$$
\begin{aligned}
A \alpha^{\prime}(t)+B \alpha(t) & =F(t) \text { for } t \geqslant 0, \\
\alpha(0) & =\gamma,
\end{aligned}
$$

where $A=\left(a_{j k}\right)$ and $B=\left(b_{j k}\right)$ are the mass and stiffness matrices whose elements are $a_{j k}=\left(\varphi_{j}, \varphi_{k}\right)$ and $b_{j k}=\left(\nabla \varphi_{j}, \nabla \varphi_{k}\right)$, respectively, where $\alpha_{j}(t)$ and $\gamma_{J}$ are the components of $u_{h}(t)$ and $v_{h}$ with respect to $\left\{\varphi_{J}\right\}$ and where $F$ is the vector with components $\left(f, \varphi_{k}\right)$.

A simple way to define the lumped mass method is to replace the matrix $A$ in (1.3) by the diagonal matrix $\bar{A}$ obtained by taking for its diagonal elements the numbers $\bar{a}_{J j}=\sum_{k-1}^{N_{h}} a_{j k}$, so that the system of ordinary differential equations becomes

$$
\bar{A} \alpha^{\prime}(t)+B \alpha(t)=F(t) \text { for } t \geqslant 0 .
$$

This procedure may also be interpreted as resulting from evaluating the first term in (1.2) by numerical quadrature: Let $\tau$ be a triangle of the triangulation $\mathscr{T}_{h}$, let $P_{\tau, j}, j=1,2,3$, be its vertices and consider the quadrature formula

$$
Q_{\tau, h}(f)=\operatorname{area} \tau \cdot \frac{1}{3} \sum_{j=1}^{3} f\left(P_{\tau, j}\right) \approx \int_{\tau} f d x
$$

We may then define an approximation of the $L_{2}$ inner product by

$$
(\psi, \chi)_{h}=\sum_{\tau \in \mathscr{F}_{h}} Q_{\tau, h}(\psi \chi),
$$

and find that (1.4) is equivalent with

$$
\left(u_{h, t}, \chi\right)_{h}+\left(\nabla u_{h}, \nabla \chi\right)=(f, \chi) \quad \forall \chi \in S_{h} .
$$

For, with $u_{h}(t)=\sum_{j=1}^{N_{h}} \alpha_{j}(t) \varphi_{j}(x)$ this follows from the trivial observation that $\left(\varphi_{j}, \varphi_{k}\right)_{h}=0$ for $j \neq k$ and

$$
\left\|\varphi_{j}\right\|_{h}^{2}=\left(\varphi_{j}, \varphi_{j}\right)_{h}=\sum_{k=1}^{N_{h}}\left(\varphi_{j}, \varphi_{k}\right) .
$$

To show the latter fact we note that $\left(\varphi_{j}, \varphi_{k}\right)$ is only nonzero for $j \neq k$ if $P_{j}$ and $P_{k}$ are neighbors and that in such a case, if $\tau$ is a triangle with $P_{j}$ and $P_{k}$ as vertices,

$$
\int_{\tau} \varphi_{j} \varphi_{k} d x=\frac{1}{12} \text { area } \tau
$$


and

$$
\int_{\tau} \varphi_{j}^{2} d x=\frac{1}{6} \operatorname{area} \tau
$$

It follows, since for each pair $P_{\jmath}, P_{k}$ there are two such triangles $\tau$, that with $D_{j}$ the union of triangles which have $P_{J}$ as a vertex,

$$
\sum_{k=1}^{N_{h}}\left(\varphi_{j}, \varphi_{k}\right)=\sum_{k \neq j}\left(\varphi_{j}, \varphi_{k}\right)+\left\|\varphi_{j}\right\|^{2}=\frac{1}{3} \operatorname{area} D_{j} .
$$

Since clearly

$$
\left\|\varphi_{J}\right\|_{h}^{2}=\sum_{\tau \in \mathscr{T}_{h}} Q_{\tau, h}\left(\varphi_{\jmath}^{2}\right)=\frac{1}{3} \text { area } D_{j}
$$

our claim (1.8) follows.

The procedure just discussed is a special case of a family of quadrature schemes analyzed in Raviart [6], where it is also shown to be the only viable such method of diagonal type. In the present case Raviart's work shows $O\left(h^{2}\right)$ order convergence in the norm in $L_{2}(\Omega)$ (cf. Theorem 1 below).

The method was applied in connection with eigenvalue problems in Tong, Pian and Bucciarelli [8], where the first term in (1.7) was interpreted instead as resulting from replacing the piecewise linear functions in (1.2) by certain piecewise constant functions. This interpretation was adopted also in Fujii [3] where it was shown that if the angles of the triangulation are all nonobtuse, then a maximum principle holds for (1.7). This was applied in Ushijima [9], [10] (cf. also Tabata [7]) to derive uniform convergence, which, except for the case of uniform triangulations, was only shown to be of first order in $h$.

In the present note we shall show a maximum-norm error estimate of essentially optimal order in $h$ which will require only that the triangulation is quasiuniform, that is, such that each triangle $\tau \in \mathscr{T}_{h}$ contains a disc of radius $c h$ with $c$ bounded below, and thus without the nonobtuseness and uniformity conditions referred to above. The basic ingredient in our proof, which follows the approach taken by Wheeler [11], [12], is a superconvergent $O\left(h^{2}\right) L_{2}$ norm error estimate for the gradient of $\theta=u_{h}-R_{h} u$, where $R_{h}$ denotes the elliptic or Ritz projection of the exact solution. Together with the "almost" Sobolev inequality

$$
\|x\|_{L_{\infty}(\Omega)} \leqslant C\left(\log \frac{1}{h}\right)^{1 / 2}\|\nabla x\|_{L_{2}(\Omega)} \quad \forall \chi \in S_{h},
$$

which is valid when the triangulation is quasiuniform, this yields a $O\left(h^{2}\left(\log \frac{1}{h}\right)^{1 / 2}\right)$ maximum norm estimate for $\theta$. Using also the well known $O\left(h^{2} \log \frac{1}{h}\right)$ maximum norm estimate for the error $\rho=R_{h} u-u$ in the elliptic projection (cf. Nitsche 
[4]), this results in a maximum norm error estimate for the lumped mass semidiscrete parabolic problem of the form

$$
\left\|u_{h}(t)-u(t)\right\|_{L_{\infty}(\Omega)} \leqslant C(t, u) h^{2} \log \frac{1}{h} \text { for } t \geqslant 0,
$$

for suitably chosen discrete initial data.

This estimate will require a certain amount of regularity of the exact solution, including, in particular, a nontrivial degree of compatibility between the data on $\partial \Omega$ for $t=0$. In the special case of the homogeneous equation, for instance, it will assume that $v \in H^{4}(\Omega)$ and $v=\Delta v=0$ on $\partial \Omega$. For this case we shall therefore also demonstrate that under the milder assumptions that $v \in H^{2}(\Omega)$ and $v=0$ on $\partial \Omega$ we have for $t>0$,

$$
\left\|\nabla\left(u_{h}(t)-R_{h} u(t)\right)\right\|_{L_{2}(\Omega)} \leqslant C h^{2} t^{-1}\|v\|_{H^{2}(\Omega)},
$$

and as a consequence

$$
\left\|u_{h}(t)-u(t)\right\|_{L_{\infty}(\Omega)} \leqslant C h^{2} \log \frac{1}{h} t^{-1}\|v\|_{H^{2}(\Omega)} .
$$

We emphasize that although our superconvergence estimates in $L_{2}$ generalize to higher dimensions, the inequality (1.9) does not, so that our maximum norm error estimates are only shown for two space dimensions.

The method of lumped masses may, of course, also be used in combination with discretization in time. One could, for instance, consider the method defined by, with $\bar{\partial}_{t}$ denoting the backward difference quotient with time step $k, U^{n}$ the approximate solution at time $t_{n}=n k$, and with $0 \leqslant \kappa \leqslant 1$,

$$
\begin{array}{cc}
\left(\bar{\partial}_{t} U^{n}, \chi\right)_{h}+\kappa\left(\nabla U^{n}, \nabla \chi\right)+(1-\kappa)\left(\nabla U^{n-1}, \nabla \chi\right)=( & \left.f\left(t_{n-1}+\kappa k\right), \chi\right) \\
& \forall \chi \in S_{h}, n=1,2, \ldots, \\
U^{0}=v_{h}, &
\end{array}
$$

or in matrix form, with $\alpha^{n}$ the vector of the components of $U^{n}$ with respect to the basis $\left\{\varphi_{J}\right\}_{1}^{N_{h}}$ and $F^{n-1+\kappa}$ the vector with components $\left(f\left(t_{n-1}+\kappa k\right), \varphi_{J}\right)$,

$$
\overline{A k}^{-1}\left(\alpha^{n}-\alpha^{n-1}\right)+\kappa B \alpha^{n}+(1-\kappa) B \alpha^{n-1}=F^{n-1+\kappa},
$$

or, since $\bar{A}+\kappa k B$ is obviously positive definite,

$$
\alpha^{n}=(\bar{A}+\kappa k B)^{-1}(\bar{A}-(1-\kappa) k B) \alpha^{n-1}+(\bar{A}+\kappa k B)^{-1} F^{n-1+\kappa} .
$$

The backward Euler method corresponds to $\kappa=1$, the Crank-Nicolson method to $\kappa=\frac{1}{2}$, and for $\kappa=0$ we have the forward Euler method which is now purely explicit since $\bar{A}$ is diagonal.

For these three different methods we first show error estimates in $L_{2}$-norm, which are of order $O\left(h^{2}+k^{2}\right)$ for the Crank-Nicolson method, and $O\left(h^{2}+k\right)$ for the forward and backward Euler methods, the former under a stability 
condition which is satisfied when the triangulations are quasiuniform and the mesh ratio $k h^{-2}$ is sufficiently small. We then also show estimates of these same orders for the gradient of $\theta^{n}=U^{n}-R_{h} u^{n}$, which as above for the semidiscrete problem yield almost optimal order error estimates in the maximum norm. The paper is completed by demonstrating some error estimates for somewhat less regular data in the case of the homogeneous equation which match our above corresponding estimates for the semidiscrete problem.

In the rest of the paper we shall denote the norm in $L_{2}(\Omega)$ by $\|\cdot\|$, that in $H^{s}(\Omega)$ by $\|\cdot\|_{s}$ and that in $L_{p}(\Omega)(p \neq 2)$ by $\|\cdot\|_{L_{p}} \cdot C$ will be different positive constants independent of $h$ and the functions involved, and not necessarily the same at different occurrences.

\section{The semidiscrete problem}

We begin our error analysis with the following lemma concerning the quadrature error

$$
\varepsilon_{h}(v, w)=(v, w)_{h}-(v, w),
$$

where $(\cdot, \cdot)_{h}$ is defined by (1.5) and (1.6).

LEMMA 1. We have for $\psi, \chi \in S_{h}$,

$$
\left|\varepsilon_{h}(\psi, \chi)\right| \leqslant C h^{2}\|\nabla \psi\| \cdot\|\nabla \chi\|,
$$

and

$$
\left|\varepsilon_{h}(\psi, \chi)\right| \leqslant C h\|\nabla \psi\| \cdot\|\chi\| .
$$

Proof. Since the quadrature formula (1.5) is exact for $f$ linear we have by transformation to a fixed reference triangle $\tau_{0}$ and using the Bramble-Hilbert lemma and the Sobolev inequality

$$
\|f\|_{L_{\infty}\left(\tau_{0}\right)} \leqslant C \sum_{|\alpha| \leqslant 2}\left\|D^{\alpha} f\right\|_{L_{1}\left(\tau_{0}\right)},
$$

that, with $h_{\tau}$ the diameter of $\tau$,

$$
\left|Q_{\tau, h}(f)-\int_{\tau} f d x\right| \leqslant C h_{\tau}^{2} \sum_{|\alpha|=2}\left\|D^{\alpha} f\right\|_{L_{1}(\tau)} .
$$

After application to $f=\psi \chi$ this implies, since both $\psi$ and $\chi$ are linear in $\tau$, that

$$
\begin{aligned}
\left|Q_{\tau, h}(\psi \chi)-\int_{\tau} \psi \chi d x\right| & \leqslant C h_{\tau}^{2} \sum_{|\alpha|=2}\left\|D^{\alpha}(\psi \chi)\right\|_{L_{1}(\tau)} \\
& \leqslant C h_{\tau}^{2}\|\nabla \psi\|_{L_{2}(\tau)}\|\nabla \chi\|_{L_{2}(\tau)} .
\end{aligned}
$$


We conclude using the Cauchy-Schwarz inequality that

$$
\left|\varepsilon_{h}(\psi, \chi)\right| \leqslant C h^{2} \sum_{\tau \in \mathscr{T}_{h}}\|\nabla \psi\|_{L_{2}(r)}\|\nabla \chi\|_{L_{2}(r)} \leqslant C h^{2}\|\nabla \psi\| \cdot\|\nabla \chi\|,
$$

which is the first of the desired inequalities. Using a local inverse estimate on $\tau$ we also have

$$
\left|Q_{\tau, h}(\psi \chi)-\int_{\tau} \psi \chi d x\right| \leqslant C h_{\tau}\|\nabla \psi\|_{L_{2}(\tau)}\|x\|_{L_{2}(\tau)},
$$

which yields the second estimate.

We shall now show the following $L_{2}$ error estimate (cf. [6]) in which we use the elliptic projection $R_{h}: H_{0}^{1}(\Omega) \rightarrow S_{h}$ defined by

$$
\left(\nabla R_{h} v, \nabla \chi\right)=(\nabla v, \nabla \chi), \quad \forall \chi \in S_{h} .
$$

Recall that

$$
\left\|R_{h} v-v\right\|+h\left\|\nabla R_{h} v-\nabla v\right\| \leqslant C h^{\jmath}\|v\|_{j} \quad \text { for } j=1,2 .
$$

THEOREM 1. Let $u_{h}$ and $u$ be the solutions of (1.7) and (1.1), respectively. We then have for $t \geqslant 0$,

$$
\left\|u_{h}(t)-u(t)\right\| \leqslant C\left\|v_{h}-R_{h} v\right\|+C h^{2}\left\{\|u(t)\|_{2}+\left(\int_{0}^{t}\left\|u_{t}\right\|_{2}^{2} d s\right)^{1 / 2}\right\} .
$$

Proof. We write

$$
u_{h}-u=\left(u_{h}-R_{h} u\right)+\left(R_{h} u-u\right)=\theta+\rho .
$$

Here by (2.1),

$$
\|\rho(t)\|=\left\|R_{h} u(t)-u(t)\right\| \leqslant C h^{2}\|u(t)\|_{2} .
$$

Turning to $\theta$ we have

$$
\begin{aligned}
& \left(\theta_{t}, \chi\right)_{h}+(\nabla \theta, \nabla \chi)=\left(u_{h, t}, \chi\right)_{h}+\left(\nabla u_{h}, \nabla \chi\right)-\left(R_{h} u_{t}, \chi\right)_{h}-\left(\nabla R_{h} u, \nabla \chi\right) \\
& \quad=(f, \chi)-\left(R_{h} u_{t}, \chi\right)_{h}-(\nabla u, \nabla \chi) \\
& \quad=\left(u_{t}, \chi\right)-\left(R_{h} u_{t}, \chi\right)_{h} \\
& \quad=-\left(\rho_{t}, \chi\right)-\varepsilon_{h}\left(R_{h} u_{t}, \chi\right)
\end{aligned}
$$

Setting $\chi=\theta$ we obtain

$$
\frac{1}{2} \frac{d}{d t}\|\theta\|_{h}^{2}+\|\nabla \theta\|^{2}=-\left(\rho_{t}, \theta\right)-\varepsilon_{h}\left(R_{h} u_{t}, \theta\right) .
$$

Here we have at once

$$
\left|\left(\rho_{t}, \theta\right)\right| \leqslant\left\|R_{h} u_{t}-u_{t}\right\| \cdot\|\theta\| \leqslant C h^{2}\left\|u_{t}\right\|_{2}\|\theta\|
$$


and, in view of Lemma 1 ,

$$
\left|\varepsilon_{h}\left(R_{h} u_{t}, \theta\right)\right| \leqslant C h^{2}\left\|\nabla R_{h} u_{t}\right\| \cdot\|\nabla \theta\| \leqslant C h^{2}\left\|\nabla u_{t}\right\| \cdot\|\nabla \theta\| .
$$

It follows that

$$
\frac{1}{2} \frac{d}{d t}\|\theta\|_{h}^{2}+\|\nabla \theta\|^{2} \leqslant C h^{2}\left\|u_{t}\right\|_{2}\|\nabla \theta\| \leqslant\|\nabla \theta\|^{2}+C h^{4}\left\|u_{t}\right\|_{2}^{2},
$$

or, after integration,

$$
\|\theta(t)\|_{h}^{2} \leqslant\|\theta(0)\|_{h}^{2}+C h^{4} \int_{0}^{t}\left\|u_{t}\right\|_{2}^{2} d s .
$$

Noting now that $\|\cdot\|_{h}$ and $\|\cdot\|$ are equivalent norms on $S_{h}$, which easily follows by considering each triangle separately, we have hence

$$
\|\theta(t)\| \leqslant C\|\theta(0)\|+C h^{2}\left(\int_{0}^{t}\left\|u_{t}\right\|_{2}^{2} d s\right)^{1 / 2},
$$

which completes the proof.

We now turn to an $L_{2}$ norm error estimate for the gradient.

THEOREM 2. With $u_{h}$ and $u$ the solution of (1.7) and (1.1) we have for $t \geqslant 0$.

$$
\left\|\nabla u_{h}(t)-\nabla u(t)\right\| \leqslant\left\|\nabla v_{h}-\nabla R_{h} v\right\|+C h\left\{\|u(t)\|_{2}+\left(\int_{0}^{t}\left\|u_{t}\right\|_{1}^{2} d s\right)^{1 / 2}\right\} .
$$

Proof. Setting this time $\chi=\theta_{t}$ in (2.2) we obtain

$$
\left\|\theta_{t}\right\|_{h}^{2}+\frac{1}{2} \frac{d}{d t}\|\nabla \theta\|^{2}=-\left(\rho_{t}, \theta_{t}\right)-\varepsilon_{h}\left(R_{h} u_{t}, \theta_{t}\right),
$$

where now

$$
\left|\left(\rho_{t}, \theta_{t}\right)\right| \leqslant\left\|R_{h} u_{t}-u_{t}\right\| \cdot\left\|\theta_{t}\right\| \leqslant C h\left\|u_{t}\right\|_{1}\left\|\theta_{t}\right\|,
$$

and by Lemma 1 ,

$$
\left|\varepsilon_{h}\left(R_{h} u_{t}, \theta_{t}\right)\right| \leqslant C h\left\|\nabla R_{h} u_{t}\right\| \cdot\left\|\theta_{t}\right\| \leqslant C h\left\|u_{t}\right\|_{1}\left\|\theta_{t}\right\| .
$$

Using again the equivalence between $\|\cdot\|_{h}$ and $\|\cdot\|$ on $S_{h}$ we conclude

$$
\left\|\theta_{t}\right\|_{h}^{2}+\frac{1}{2} \frac{d}{d t}\|\nabla \theta\|^{2} \leqslant C h\left\|u_{t}\right\|_{1}\left\|\theta_{t}\right\|_{h} \leqslant\left\|\theta_{t}\right\|_{h}^{2}+C h^{2}\left\|u_{t}\right\|_{1}^{2},
$$

whence

$$
\|\nabla \theta(t)\|^{2} \leqslant\|\nabla \theta(0)\|^{2}+C h^{2} \int_{0}^{t}\left\|u_{t}\right\|_{1}^{2} d s .
$$

Together with

$$
\|\nabla \rho(t)\|=\left\|\nabla R_{h} u(t)-\nabla u(t)\right\| \leqslant C h\|u(t)\|_{2},
$$

this completes the proof. 
A natural choice for discrete initial data in Theorems 1 and 2 is $v_{h}=R_{h} v$ but also any other optimal order approximation to $v$ in $S_{h}$ produces optimal order error estimates for the parabolic problem.

We shall now show that a slight modification of the above proof yields a superconvergent $O\left(h^{2}\right)$ estimate for $\nabla \theta$ similar to the case of the standard Galerkin method (1.2).

LEMMA 2. Let $u_{h}$ and $u$ be the solutions of (1.7) and (1.1). Then for each $T>0$ there is a constant $C=C_{T}$ such that for $\theta=u_{h}-R_{h} u$ and $0 \leqslant t \leqslant T$,

$$
\|\nabla \theta(t)\| \leqslant\|\nabla \theta(0)\|+C h^{2}\left\{\left\|u_{t}(t)\right\|_{1}+\left(\int_{0}^{t}\left(\left\|u_{t}\right\|_{2}^{2}+\left\|u_{t t}\right\|_{1}^{2}\right) d s\right)^{1 / 2}\right\} .
$$

Proof. It suffices to consider the case $\theta(0)=0$. For the solution $\tilde{u}_{h}$ of the homogeneous equation with initial data $\tilde{u}_{h}(0)=\theta(0)$ satisfies

$$
\left\|\tilde{u}_{h, t}\right\|_{h}^{2}+\frac{1}{2} \frac{d}{d t}\left\|\nabla \tilde{u}_{h}\right\|^{2}=0,
$$

and hence

$$
\left\|\nabla \tilde{u}_{h}(t)\right\| \leqslant\left\|\nabla \tilde{u}_{h}(0)\right\|=\|\nabla \theta(0)\| .
$$

We have as before (2.4) which we now write in the form

$$
\left\|\theta_{t}\right\|_{h}^{2}+\frac{1}{2} \frac{d}{d t}\|\nabla \theta\|^{2}=-\left(\rho_{t}, \theta_{t}\right)-\frac{d}{d t} \varepsilon_{h}\left(R_{h} u_{t}, \theta\right)+\varepsilon_{h}\left(R_{h} u_{t t}, \theta\right) .
$$

Here

$$
\left|\left(\rho_{t}, \theta_{t}\right)\right| \leqslant\left\|\rho_{t}\right\| \cdot\left\|\theta_{t}\right\| \leqslant C h^{2}\left\|u_{t}\right\|_{2}\left\|\theta_{t}\right\|_{h} \leqslant C h^{4}\left\|u_{t}\right\|_{2}^{2}+\left\|\theta_{t}\right\|_{h}^{2} .
$$

Further, by Lemma 1 ,

$$
\left|\varepsilon_{h}\left(R_{h} u_{t}, \theta\right)\right| \leqslant C h^{2}\left\|\nabla R_{h} u_{t}\right\| \cdot\|\nabla \theta\| \leqslant C h^{4}\left\|u_{t}\right\|_{1}^{2}+\frac{1}{4}\|\nabla \theta\|^{2},
$$

and similarly with $u_{t}$ replaced by $u_{t}$. By integration of (2.5) we therefore obtain

$$
\|\nabla \theta(t)\|^{2} \leqslant C h^{4}\left\{\left\|u_{t}(t)\right\|_{1}^{2}+\int_{0}^{t}\left(\left\|u_{t}\right\|_{2}^{2}+\left\|u_{t t}\right\|_{1}^{2}\right) d s\right\}+\int_{0}^{t}\|\nabla \theta\|^{2} d s .
$$

The result now follows by Gronwall's lemma.

As an application of the lemma we prove the following maximum-norm error estimate:

THEOREM 3. Assume that the triangulation $\mathscr{T}_{h}$ is quasiuniform and let $u_{h}$ and $u$ be the solutions of (1.7) and (1.1), with $v_{h}=R_{h} v$. Then for each $T>0$ there is a 
constant $C=C_{T}$ such that for $0 \leqslant t \leqslant T$,

$$
\begin{aligned}
\left\|u_{h}(t)-u(t)\right\|_{L_{\infty}} \leqslant C h^{2} \log \frac{1}{h}\left\{\|u(t)\|_{w_{\infty}^{2}(\Omega)}+\left\|u_{t}(t)\right\|_{1}\right. & \\
& \left.+\left(\int_{0}^{t}\left(\left\|u_{t}\right\|_{2}^{2}+\left\|u_{t t}\right\|_{1}^{2}\right) d s\right)^{1 / 2}\right\} .
\end{aligned}
$$

Proof. By a known maximum-norm error estimate for the elliptic problem (cf. Nitsche [4]) we have

$$
\|\rho(t)\|_{L_{\infty}} \leqslant\left\|R_{h} u(t)-u(t)\right\|_{L_{\infty}} \leqslant C h^{2} \log \frac{1}{h}\|u(t)\|_{W_{\infty}^{2}(\Omega)} .
$$

In order to bound $\theta(t)$ we note that

$$
\|\chi\|_{L_{\infty}} \leqslant C\left(\log \frac{1}{h}\right)^{1 / 2}\|\nabla \chi\| \quad \forall \chi \in S_{h} .
$$

In fact, by Sobolev's lemma there is a constant $C$ independent of $p>2$ such that

$$
\|\chi\|_{L_{p}} \leqslant C p^{1 / 2}\|\nabla \chi\| \quad \forall \chi \in S_{h} .
$$

On the other hand, since $\mathscr{T}_{h}$ is quasiuniform, we have the inverse estimate

$$
\|\chi\|_{L_{\infty}} \leqslant C h^{-2 / p}\|\chi\|_{L_{p}} \quad \forall \chi \in S_{h} .
$$

Choosing $p=\log (1 / h)$ these inequalities show (2.7). Application to $\theta(t)$ and invoking Lemma 2 now yields

$$
\begin{aligned}
\|\theta(t)\|_{L_{\infty}} & \leqslant C\left(\log \frac{1}{h}\right)^{1 / 2}\|\nabla \theta\| \\
& \leqslant C h^{2} \log \frac{1}{h}\left\{\left\|u_{t}(t)\right\|_{1}+\left(\int_{0}^{t}\left(\left\|u_{t}\right\|_{2}^{2}+\left\|u_{t t}\right\|_{1}^{2}\right) d s\right)^{1 / 2}\right\},
\end{aligned}
$$

thus completing the proof.

We observe that our above error analysis requires more regularity of the solution than known error estimates for the standard Galerkin method. For instance, for the standard method (1.2) with $v_{h}=R_{h} v$, applied to the homogeneous equation $(f=0)$ one has (cf. [2])

$$
\left\|u_{h}(t)-u(t)\right\| \leqslant C h^{2}\|v\|_{2} \text { if } v=0 \text { on } \partial \Omega,
$$

whereas for the lumped mass method, Theorem 1 only yields

$$
\left\|u_{h}(t)-u(t)\right\| \leqslant C h^{2}\|v\|_{3} \quad \text { if } v=\Delta v=0 \text { on } \partial \Omega .
$$

To prove the latter estimate, we recall that with $\{\lambda,\}_{1}^{\infty}$ and $\left\{\varphi_{J}\right\}_{1}^{\infty}$ the eigenvalues and eigenfunctions of $-\Delta$ with homogeneous Dirichlet boundary conditions, the norm $\|v\|_{s}$ is equivalent to $\left(\sum_{j=1}^{\infty} \lambda_{j}^{s}\left(v, \varphi_{j}\right)^{2}\right)^{1 / 2}$ for functions which satisfy the 
boundary conditions $\Delta^{\prime} v=0$ on $\partial \Omega$ for $j<s / 2$. Hence one has for the solution of the homogeneous equation

$$
\|u(t)\|_{2}^{2} \leqslant C \sum_{j} \lambda_{j}^{2} e^{-2 \lambda, t}\left(v, \varphi_{j}\right)^{2} \leqslant C\|v\|_{2}^{2}
$$

and

$$
\int_{0}^{t}\left\|u_{t}\right\|_{2}^{2} d s \leqslant C \int_{0}^{l} \sum_{j} \lambda_{j}^{4} e^{-2 \lambda_{j} s}\left(v, \varphi_{j}\right)^{2} d s \leqslant C \sum_{j} \lambda_{j}^{3}\left(v, \varphi_{j}\right)^{2} \leqslant C\|v\|_{3}^{2},
$$

which gives (2.8). Similarly Lemma 2 shows

$$
\|\nabla \theta(t)\| \leqslant C h^{2}\|v\|_{4} \quad \text { if } v=\Delta v=0 \text { on } \partial \Omega,
$$

whereas the standard Galerkin method only requires $v$ to have three derivatives. The boundary condition $\Delta v=0$ on $\partial \Omega$ in (2.8) and (2.9) is particularly undesirable in applications.

For the standard Galerkin method, on the other hand, it is known that the convergence is of optimal order for $t$ positive, even if $v$ is only in $L_{2}$, or

$$
\left\|u_{h}(t)-u(t)\right\| \leqslant C h^{2} t^{-1}\|v\| .
$$

We shall now demonstrate a weaker result of similar nature in the present situation. It will reduce the regularity assumptions for optimal order convergence for $t$ positive to $v \in H^{2}(\Omega)$ and $v=0$ on $\partial \Omega$, and thus eliminate the requirement that $\Delta v=0$ on $\partial \Omega$.

LEMMA 3. Consider the homogeneous equation $(f=0)$ and let $\theta=u_{h}-R_{h} u$. Then for each $T>0$ there is a constant $C=C_{T}$ such that if $\theta(0)=0$ then for $0<t \leqslant T$ and $v \in H^{2}(\Omega)$ with $v=0$ on $\partial \Omega$,

$$
\|\theta(t)\| \leqslant C h^{2} t^{-1 / 2}\|v\|_{2}
$$

and

$$
\|\nabla \theta\| \leqslant C h^{2} t^{-1}\|v\|_{2} .
$$

Proof. Multiplying (2.3) by $t$ we have

$$
\frac{1}{2} \frac{d}{d t}\left(t\|\theta\|_{h}^{2}\right)+t\|\nabla \theta\|^{2}=-t\left(\rho_{t}, \theta\right)-t \varepsilon_{h}\left(R_{h} u_{t}, \theta\right)+\frac{1}{2}\|\theta\|_{h}^{2} .
$$

Hence by integration and routine estimates, using Lemma 1 and (2.1),

$$
\begin{aligned}
t\|\theta(t)\|_{h}^{2}+\int_{0}^{t} s\|\nabla \theta\|^{2} d s & \leqslant C h^{4} \int_{0}^{t}\left(s^{2}\left\|u_{t}\right\|_{2}^{2}+s\left\|u_{t}\right\|_{1}^{2}\right) d s+C \int_{0}^{t}\|\theta\|_{h}^{2} d s \\
& \leqslant C h^{4}\|v\|_{1}^{2}+C \int_{0}^{t}\|\theta\|_{h}^{2} d s .
\end{aligned}
$$


In order to bound the latter integral we set $\Theta(t)=\int_{0}^{t} \Theta(s) d s$ and integrate the error equation (2.2) from 0 to $t$ to obtain

$$
(\theta, \chi)_{h}+(\nabla \Theta, \nabla \chi)=(\rho(0)-\rho(t), \chi)-\varepsilon_{h}\left(R_{h}(u(t)-v), \chi\right) \quad \forall \chi \in S_{h} .
$$

Setting $\chi=\theta=\Theta_{,}$this yields

$$
\begin{aligned}
\|\theta\|_{h}^{2}+\frac{1}{2} \frac{d}{d t}\|\nabla \Theta\|^{2}= & (\rho(0)-\rho(t), \theta)-\frac{d}{d t} \varepsilon_{h}\left(R_{h}(u(t)-v), \Theta\right) \\
& +\varepsilon_{h}\left(R_{h} u_{t}, \Theta\right),
\end{aligned}
$$

and hence

$$
\begin{aligned}
\int_{0}^{t}\|\theta\|_{h}^{2} d s+\|\nabla \Theta\|^{2} \leqslant & C h^{4} \int_{0}^{t}\left(\|u(s)\|_{2}^{2}+\|v\|_{2}^{2}\right) d s+C h^{4}\left\|\nabla R_{h}(u(t)-v)\right\|^{2} \\
& +C h^{4} \int_{0}^{t}\left\|\nabla R_{h} u_{t}\right\|^{2} d s+\int_{0}^{t}\|\nabla \Theta\|^{2} d s \\
\leqslant & C h^{4}\|v\|_{2}^{2}+\int_{0}^{t}\|\nabla \Theta\|^{2} d s,
\end{aligned}
$$

so that, using also Gronwall's lemma, for $t \leqslant T$,

$$
\int_{0}^{t}\|\theta\|_{h}^{2} d s \leqslant C h^{4}\|v\|_{2}^{2} \text {. }
$$

Together with (2.10) this proves the first estimate of the lemma.

In order to show the estimate for $\nabla \theta$ we multiply (2.5) by $t^{2}$ and obtain

$$
\begin{aligned}
\frac{1}{2} \frac{d}{d t}\left(t^{2}\|\nabla \theta\|^{2}\right) \leqslant & -\frac{d}{d t}\left(t^{2} \varepsilon_{h}\left(R_{h} u_{t}, \theta\right)\right)+C t^{2}\left\|\rho_{t}\right\|^{2} \\
& +t^{2} \varepsilon_{h}\left(R_{h} u_{t}, \theta\right)+2 t \varepsilon_{h}\left(R_{h} u_{t}, \theta\right)+t\|\nabla \theta\|^{2} .
\end{aligned}
$$

By integration and obvious estimates this yields

$$
\begin{aligned}
t^{2}\|\nabla \theta(t)\|^{2} \leqslant & C t^{2} h^{2}\left\|\nabla R_{h} u_{t}(t)\right\| \cdot\|\nabla \theta(t)\|+C h^{4} \int_{0}^{t} s^{2}\left\|u_{t}\right\|_{2}^{2} d s \\
& +C h^{4} \int_{0}^{t}\left(s^{3}\left\|\nabla R_{h} u_{t}\right\|^{2}+s\left\|\nabla R_{h} u_{t}\right\|^{2}\right) d s+C \int_{0}^{t} s\|\nabla \theta\|^{2} d s,
\end{aligned}
$$

or

$$
\begin{aligned}
t^{2}\|\nabla \theta(t)\|^{2} \leqslant & C h^{4}\left\{t^{2}\left\|\nabla u_{t}(t)\right\|^{2}+\int_{0}^{t}\left(s^{3}\left\|u_{t}\right\|_{1}^{2}+s^{2}\left\|u_{t}\right\|_{2}^{2}+s\left\|u_{t}\right\|_{1}^{2}\right) d s\right\} \\
& +C \int_{0}^{t} s\|\nabla \theta\|^{2} d s \\
\leqslant & C h^{4}\|v\|^{2}+C \int_{0}^{t} s\|\nabla \theta\|^{2} d s,
\end{aligned}
$$

and hence, using our above estimates (2.10) and (2.11) we have, since $t \leqslant T$,

$$
t^{2}\|\nabla \theta(t)\|^{2} \leqslant C h^{4}\|v\|_{2}^{2},
$$

which completes the proof. 
As a consequence we have the following:

THEOREM 4. Let $u_{h}$ and $u$ be the solutions of (1.7) and (1.1), respectively, with $f=0$, and let $v_{h}=R_{h} v$. Then for each $T>0$ there is a constant $C=C_{T}$ such that for $0<t \leqslant T$ and $v=0$ on $\partial \Omega$,

$$
\left\|u_{h}(t)-u(t)\right\| \leqslant C h^{2} t^{-1 / 2}\|v\|_{2},
$$

and, if the triangulation $\mathscr{T}_{h}$ is quasiuniform,

$$
\left\|u_{h}(t)-u(t)\right\|_{L_{\infty}} \leqslant C h^{2} \log \frac{1}{h} \cdot t^{-1}\|v\|_{2} .
$$

Proof. We have by (2.1) for $\rho=R_{h} u-u$,

$$
\|\rho(t)\| \leqslant C h^{2}\|u(t)\|_{2} \leqslant C h^{2}\|v\|_{2} .
$$

Also, using (2.6), a Sobolev inequality, and the spectral norm discussed above,

$$
\begin{aligned}
\|\rho(t)\|_{L_{\infty}} & \leqslant C h^{2} \log \frac{1}{h}\|u(t)\|_{w_{\infty}^{2}(\Omega)} \leqslant C h^{2} \log \frac{1}{h}\|u(t)\|_{4} \\
& \leqslant C h^{2} \log \frac{1}{h} \cdot t^{-1}\|v\|_{2} .
\end{aligned}
$$

Together with the estimates of Lemma 3 these bounds show our assertions.

\section{Totally discrete schemes}

We shall begin our discussion of totally discrete schemes by showing an $L_{2}$ norm error estimate for the lumped mass backward Euler method

$$
\begin{gathered}
\left(\bar{\partial}_{t} U^{n}, \chi\right)_{h}+\left(\nabla U^{n}, \nabla \chi\right)=\left(f^{n}, \chi\right) \quad \forall \chi \in S_{h}, \\
U^{0}=v_{h}
\end{gathered}
$$

where $U^{n} \in S_{h}$ is the approximate solution at $t_{n}=n k$ and $f^{n}=f\left(t_{n}\right)$. For simplicity we shall always choose $v_{h}=R_{h} v$ for discrete initial data below, where $R_{h}$ denotes the elliptic projection defined earlier; the modifications needed for other natural choices are trivial.

THEOREM 5. Let $u$ and $U^{n}$ be the solutions of (1.1) and (3.1) with $v_{h}=R_{h} v$. Then for $t_{n}=n k \geqslant 0$,

$$
\begin{aligned}
\left\|U^{n}-u\left(t_{n}\right)\right\| \leqslant & C h^{2}\left\{\left\|u\left(t_{n}\right)\right\|_{2}+\left(\int_{0}^{t_{n}}\left\|u_{t}\right\|_{2}^{2} d s\right)^{1 / 2}\right\} \\
& +C k\left(\int_{0}^{t_{n}}\left\|u_{t t}\right\|^{2} d s\right)^{1 / 2} .
\end{aligned}
$$


Proof. Setting as usual, with $u^{n}=u\left(t_{n}\right)$ etc.,

$$
U^{n}-u^{n}=\left(U^{n}-R_{h} u^{n}\right)+\left(R_{h} u^{n}-u^{n}\right)=\theta^{n}+\rho^{n},
$$

we have

$$
\left\|\rho^{n}\right\| \leqslant C h^{2}\left\|u^{n}\right\|_{2}
$$

For $\theta^{n}$ we obtain

$$
\begin{aligned}
\left(\bar{\partial}_{t} \theta^{n}, \chi\right)_{h}+\left(\nabla \theta^{n}, \nabla \chi\right) \\
\quad=\left(\bar{\partial}_{t} U^{n}, \chi\right)_{h}+\left(\nabla U^{n}, \nabla \chi\right)-\left(\bar{\partial}_{t} R_{h} u^{n}, \chi\right)_{h}-\left(\nabla R_{h} u^{n}, \nabla \chi\right) \\
\quad=\left(f^{n}, \chi\right)-\left(\bar{\partial}_{t} R_{h} u^{n}, \chi\right)_{h}-\left(\nabla u^{n}, \nabla \chi\right) \\
\quad=\left(u_{t}^{n}, \chi\right)-\left(\bar{\partial}_{t} R_{h} u^{n}, \chi\right)_{h} \\
\quad=\left(u_{t}^{n}-\bar{\partial}_{t} u^{n}, \chi\right)+\left(\bar{\partial}_{t} u^{n}-\bar{\partial}_{t} R_{h} u^{n}, \chi\right)-\varepsilon_{h}\left(\bar{\partial}_{t} R_{h} u^{n}, \chi\right) \\
\quad=T_{1}^{n}+T_{2}^{n}+T_{3}^{n} .
\end{aligned}
$$

Here

$$
\begin{aligned}
\left|T_{2}^{n}\right| & \leqslant\left\|\left(I-R_{h}\right) \bar{\partial}_{t} u^{n}\right\| \cdot\|\chi\| \leqslant C h^{2}\left\|\bar{\partial}_{t} u^{n}\right\|_{2}\|\chi\| \\
& \leqslant C h^{2}\left\|k^{-1} \int_{t_{n-1}}^{t_{n}} u_{t} d s\right\|_{2}\|x\| \leqslant C h^{2} k^{-1 / 2}\left(\int_{t_{n-1}}^{t_{n}}\left\|u_{t}\right\|_{2}^{2} d s\right)^{1 / 2}\|x\| .
\end{aligned}
$$

Further, using Lemma 1,

$$
\begin{aligned}
\left|T_{3}^{n}\right| & \leqslant C h^{2}\left\|\nabla R_{h} \bar{\partial}_{t} u^{n}\right\| \cdot\|\nabla \chi\| \leqslant C h^{2}\left\|\bar{\partial}_{t} \nabla u^{n}\right\| \cdot\|\nabla \chi\| \\
& \leqslant C h^{2} k^{-1 / 2}\left(\int_{t_{n-1}}^{t_{n}}\left\|u_{t}\right\|_{1}^{2} d s\right)^{1 / 2}\|\nabla \chi\|
\end{aligned}
$$

and for the contribution from the discretization in time,

$$
\begin{aligned}
\left|T_{1}^{n}\right| & \leqslant\left\|u_{t}^{n}-\bar{\partial}_{t} u^{n}\right\| \cdot\|\chi\|=\left\|k^{-1} \int_{t_{n-1}}^{t_{n}}\left(s-t_{n-1}\right) u_{t}(s) d s\right\| \cdot\|\chi\| \\
& \leqslant C k^{1 / 2}\left(\int_{t_{n-1}}^{t_{n}}\left\|u_{t}\right\|^{2} d s\right)^{1 / 2}\|\chi\| .
\end{aligned}
$$

Choosing $\chi=\theta^{n}$ in (3.3) we hence find after some manipulation, since $\|\chi\| \leqslant$ $C\|\nabla \times\|$,

$$
\begin{aligned}
\frac{1}{2 k}\left(\left\|\theta^{n}\right\|_{h}^{2}-\left\|\theta^{n-1}\right\|_{h}^{2}\right) & +\frac{1}{2 k}\left\|\theta^{n}-\theta^{n-1}\right\|_{h}^{2}+\left\|\nabla \theta^{n}\right\|^{2} \\
& \leqslant\left\|\nabla \theta^{n}\right\|^{2}+C h^{4} k^{-1} \int_{t_{n-1}}^{t_{n}}\left\|u_{t}\right\|_{2}^{2} d s+C k \int_{t_{n-1}}^{t_{n}}\left\|u_{t i}\right\|^{2} d s,
\end{aligned}
$$


whence

$$
\left\|\theta^{n}\right\|_{h}^{2} \leqslant\left\|\theta^{n-1}\right\|_{h}^{2}+C h^{4} \int_{t_{n-1}}^{t_{n}}\left\|u_{t}\right\|_{2}^{2} d s+C k^{2} \int_{t_{n-1}}^{t_{n}}\left\|u_{t t}\right\|^{2} d s,
$$

and, since $\theta^{0}=0$,

$$
\left\|\theta^{n}\right\|_{h}^{2} \leqslant C h^{4} \int_{0}^{t_{n}}\left\|u_{t}\right\|_{2}^{2} d s+C k^{2} \int_{0}^{t_{n}}\left\|u_{t t}\right\|^{2} d s .
$$

Recalling that $\|\cdot\|_{h}$ and $\|\cdot\|$ are equivalent this concludes the proof.

We shall now derive the following superconvergent order $L_{2}$ estimate for the gradient of $U^{n}-R_{h} u\left(t_{n}\right)$.

LEMMA 4. Let $u$ and $U^{n}$ be the solutions of (1.1) and (3.1) with $v_{h}=R_{h} v$. Then for $\theta^{n}=U^{n}-R_{h} u\left(t_{n}\right)$ we have for $0 \leqslant t_{n}=n k \leqslant T$, with $C=C_{T}$,

$$
\begin{aligned}
\left\|\nabla \theta^{n}\right\| \leqslant & C h^{2}\left\{\left\|u_{t}(0)\right\|_{1}^{2}+\left(\int_{0}^{t_{n}}\left(\left\|u_{t}\right\|_{2}^{2}+\left\|u_{t t}\right\|_{1}^{2}\right) d s\right)^{1 / 2}\right\} \\
& +C k\left(\int_{0}^{t_{n}}\left\|u_{t t}\right\|^{2} d s\right)^{1 / 2} .
\end{aligned}
$$

Proof. We use again (3.3), now with $\chi=\bar{\partial}_{t} \theta^{n}$ and obtain

$$
\left\|\bar{\partial}_{t} \theta^{n}\right\|_{h}^{2}+\frac{1}{2 k}\left(\left\|\nabla \theta^{n}\right\|^{2}-\left\|\nabla \theta^{n-1}\right\|^{2}\right)+\frac{k}{2}\left\|\nabla \bar{\partial}_{t} \theta^{n}\right\|^{2}=T_{1}^{n}+T_{2}^{n}+T_{3}^{n} .
$$

Here, by (3.4) and (3.6),

$$
\left|T_{1}^{n}\right|+\left|T_{2}^{n}\right| \leqslant C h^{4} k^{-1} \int_{t_{n-1}}^{t_{n}}\left\|u_{t}\right\|_{2}^{2} d s+C k \int_{t_{n-1}}^{t_{n}}\left\|u_{t}\right\|^{2} d s+\left\|\bar{\partial}_{t} \theta^{n}\right\|_{h}^{2} .
$$

By summation we thus obtain from (3.7) since $\theta^{0}=0$,

$$
\left\|\nabla \theta^{n}\right\|^{2} \leqslant C h^{4} \int_{0}^{t_{n}}\left\|u_{t}\right\|_{2}^{2} d s+C k^{2} \int_{0}^{t_{n}}\left\|u_{t}\right\|^{2} d s+2 k \sum_{j=1}^{n} T_{3}^{j}
$$

We now note that

$$
T_{3}^{j}=-\varepsilon_{h}\left(\bar{\partial}_{t} R_{h} u^{j}, \bar{\partial}_{t} \theta^{j}\right)=-\bar{\partial}_{t} \varepsilon_{h}\left(\bar{\partial}_{t} R_{h} u^{j}, \theta^{j}\right)+\varepsilon_{h}\left(\bar{\partial}_{t}^{2} R_{h} u^{j}, \theta^{j-1}\right),
$$

whence

$$
2 k \sum_{j=1}^{n} T_{3}^{j}=-2 \varepsilon_{h}\left(\bar{\partial}_{t} R_{h} u^{n}, \theta^{n}\right)+2 k \sum_{j=1}^{n} \varepsilon_{h}\left(\bar{\partial}_{t}^{2} R_{h} u^{j}, \theta^{j-1}\right)
$$


Here

$$
\begin{aligned}
\left|\varepsilon_{h}\left(\bar{\partial}_{t} R_{h} u^{n}, \theta^{n}\right)\right| & \leqslant C h^{2}\left\|\bar{\partial}_{t} u^{n}\right\|_{1}\left\|\nabla \theta^{n}\right\| \\
& \leqslant C h^{2} k^{-1} \int_{t_{n-1}}^{t_{n}}\left\|u_{t}\right\|_{1} d s\left\|\nabla \theta^{n}\right\| \\
& \leqslant C h^{4}\left(k^{-1} \int_{t_{n-1}}^{t_{n}}\left\|u_{t}\right\|_{1} d s\right)^{2}+\frac{1}{2}\left\|\nabla \theta^{n}\right\|^{2} \\
& \leqslant C h^{4}\left\{\left\|u_{t}(0)\right\|_{1}^{2}+\int_{0}^{t_{n}}\left\|u_{t}\right\|_{1}^{2} d s\right\}+\frac{1}{2}\left\|\nabla \theta^{n}\right\|,
\end{aligned}
$$

and similarly

$$
k \sum_{j=1}^{n}\left|\varepsilon_{h}\left(\bar{\partial}_{i}^{2} R_{h} u^{j}, \theta^{j-1}\right)\right| \leqslant C h^{4} \int_{0}^{t_{h}}\left\|u_{t u}\right\|_{1}^{2} d s+C k \sum_{j=1}^{n}\left\|\nabla \theta^{j-1}\right\|^{2},
$$

so that altogether we have now shown

$$
\begin{aligned}
\left\|\nabla \theta^{n}\right\|^{2} \leqslant & C h^{4}\left\{\left\|u_{t}(0)\right\|_{1}^{2}+\int_{0}^{t_{n}}\left(\left\|u_{t}\right\|_{2}^{2}+\left\|u_{t t}\right\|_{1}^{2}\right) d s\right\} \\
& +C k^{2} \int_{0}^{t_{n}}\left\|u_{t t}\right\|^{2} d s+C k \sum_{j=1}^{n-1}\left\|\nabla \theta^{\jmath}\right\|^{2} .
\end{aligned}
$$

This shows the desired result by the discrete Gronwall's lemma.

Using the almost Sobolev inequality (2.7) and the elliptic error estimate (2.6) we conclude at once the following maximum norm estimate:

THEOREM 6. Assume that the triangulation $\mathscr{T}_{h}$ is quasiuniform and let $u$ and $U^{n}$ be the solutions of (1.1) and (3.1) with $v_{h}=R_{h} v$. Then for $0 \leqslant t_{n}=n k \leqslant T$, with $C=C_{T}$,

$$
\begin{aligned}
\left\|U^{n}-u\left(t_{n}\right)\right\|_{L_{\infty}} \leqslant & C h^{2} \log \frac{1}{h}\left\|u\left(t_{n}\right)\right\|_{W_{\infty}^{2}(\Omega)} \\
& +C h^{2}\left(\log \frac{1}{h}\right)^{1 / 2}\left\{\left\|u_{t}(0)\right\|_{1}+\left(\int_{0}^{t_{n}}\left(\left\|u_{t}\right\|_{2}^{2}+\left\|u_{t}\right\|_{1}^{2}\right) d s\right)^{1 / 2}\right\} \\
& +C k\left(\log \frac{1}{h}\right)^{1 / 2}\left(\int_{0}^{t_{n}}\left\|u_{t}\right\|^{2} d s\right)^{1 / 2} .
\end{aligned}
$$

We now turn to the second order in time lumped mass Crank-Nicolson scheme

$$
\left(\bar{\partial}_{l} U^{n}, \chi\right)_{h}+\left(\nabla \frac{U^{n}+U^{n-1}}{2}, \nabla \chi\right)=\left(f^{n-1 / 2}, \chi\right) \quad \forall \chi \in S_{h} .
$$


We have now:

THEOREM 7. Let $u$ and $U^{n}$ denote the solutions of (1.1) and (3.11) with $U^{0}=R_{h} v$. Then for $t_{n}=n k \geqslant 0$,

$$
\begin{aligned}
\left\|U^{n}-u\left(t_{n}\right)\right\| \leqslant & C h^{2}\left\{\left\|u\left(t_{n}\right)\right\|_{2}+\left(\int_{0}^{t_{n}}\left\|u_{t}\right\|_{2}^{2} d s\right)^{1 / 2}\right\} \\
& +C k^{2}\left(\int_{0}^{t_{n}}\left(\left\|u_{t t}\right\|_{1}^{2}+\left\|u_{t t}\right\|^{2}\right) d s\right)^{1 / 2} .
\end{aligned}
$$

If the triangulation $\mathscr{T}_{h}$ is quasiuniform we have for $0 \leqslant t_{n}=n k \leqslant T$, with $C=C_{T}$,

$$
\begin{aligned}
\left\|U^{n}-u\left(t_{n}\right)\right\|_{L_{\infty}} \leqslant & C h^{2} \log \frac{1}{h}\left\|u\left(t_{n}\right)\right\|_{W_{\infty}^{2}(\Omega)} \\
& +C h^{2}\left(\log \frac{1}{h}\right)^{1 / 2}\left\{\left\|u_{t}(0)\right\|_{1}+\left(\int_{0}^{t_{n}}\left(\left\|u_{t}\right\|_{2}^{2}+\left\|u_{t u}\right\|_{1}^{2}\right) d s\right)^{1 / 2}\right\} \\
& +C k^{2}\left(\log \frac{1}{h}\right)^{1 / 2}\left(\int_{0}^{t_{n}}\left(\left\|u_{t t}\right\|_{2}^{2}+\left\|u_{t u}\right\|^{2}\right) d s\right)^{1 / 2}
\end{aligned}
$$

Proof. Writing again $U^{n}-u^{n}=\theta^{n}+\rho^{n}$ we have as before the estimate (3.2) for $\rho^{n}$. We have this time for $\theta^{n}$,

$$
\begin{aligned}
\left(\bar{\partial}_{t} \theta^{n}, \chi\right)_{h}+(\nabla & \left.\left(\theta^{n}+\theta^{n-1}\right) / 2, \nabla \chi\right)=\left(\bar{\partial}_{t} U^{n}, \chi\right)_{h}+\left(\nabla\left(U^{n}+U^{n-1}\right) / 2, \nabla \chi\right) \\
& -\left(\bar{\partial}_{t} R_{h} u^{n}, \chi\right)_{h}-\left(\nabla\left(R_{h} u^{n}+R_{h} u^{n-1}\right) / 2, \nabla \chi\right) \\
= & \left(f^{n-1 / 2}, \chi\right)-\left(\bar{\partial}_{t} R_{h} u^{n}, \chi\right)_{h}-\left(\nabla\left(u^{n}+u^{n-1}\right) / 2, \nabla \chi\right) \\
= & \left(u_{t}^{n-1 / 2}, \chi\right)+\left(\nabla u^{n-1 / 2}, \nabla \chi\right) \\
& -\left(\bar{\partial}_{t} R_{h} u^{n}, \chi\right)_{h}-\left(\nabla\left(u^{n}+u^{n-1}\right) / 2, \nabla \chi\right) \\
= & \left(\nabla\left(u^{n-1 / 2}-\left(u^{n}+u^{n-1}\right) / 2\right), \nabla \chi\right) \\
& +\left(u_{t}^{n-1 / 2}-\bar{\partial}_{t} u^{n}, \chi\right)+\left(\bar{\partial}_{t} u^{n}-\bar{\partial}_{t} R_{h} u^{n}, \chi\right)-\varepsilon_{h}\left(\bar{\partial}_{t} R_{h} u^{n}, \chi\right) \\
= & \sum_{j=1}^{4} T_{j}^{n} .
\end{aligned}
$$

Here

$$
\begin{aligned}
\left|T_{1}^{n}\right| & \leqslant\left\|u^{n-1 / 2}-\left(u^{n}+u^{n-1}\right) / 2\right\|_{1}\|\nabla x\| \\
& \leqslant C k \int_{t_{n-1}}^{t_{n}}\left\|u_{t}\right\|_{1} d s\|\nabla x\| \\
& \leqslant C k^{3 / 2}\left(\int_{t_{n-1}}^{t_{n}}\left\|u_{t t}\right\|_{1}^{2} d s\right)^{1 / 2}\|\nabla x\|
\end{aligned}
$$


and similarly

$$
\left|T_{2}^{n}\right| \leqslant\left\|u_{t}^{n-1 / 2}-\bar{\partial}_{t} u^{n}\right\| \cdot\|\chi\| \leqslant C k^{3 / 2}\left(\int_{t_{n-1}}^{t_{n}}\left\|u_{t u t}\right\|^{2}\right)^{1 / 2}\|\chi\| .
$$

As in (3.4),

$$
\left|T_{3}^{n}\right| \leqslant C h^{2} k^{-1 / 2}\left(\int_{t_{n-1}}^{t_{n}}\left\|u_{t}\right\|_{2}^{2} d s\right)^{1 / 2}\|\chi\|
$$

and as in (3.5)

$$
\left|T_{4}^{n}\right| \leqslant C h^{2} k^{-1 / 2}\left(\int_{t_{n-1}}^{t_{n}}\left\|u_{t}\right\|_{1}^{2} d s\right)^{1 / 2}\|\nabla \chi\| .
$$

Setting $\chi=\theta^{n}+\theta^{n-1}$ we now have from (3.14),

$$
\begin{aligned}
& k^{-1}\left(\left\|\theta^{n}\right\|_{h}^{2}-\left\|\theta^{n-1}\right\|_{h}^{2}\right)+\frac{1}{2}\left\|\nabla\left(\theta^{n}+\theta^{n-1}\right)\right\|^{2} \\
& \leqslant C\left\{h^{2} k^{-1 / 2}\left(\int_{t_{n-1}}^{t_{n}}\left\|u_{t}\right\|_{2}^{2} d s\right)^{1 / 2}\right. \\
& \left.\quad+k^{3 / 2}\left(\int_{t_{n-1}}^{t_{n}}\left(\left\|u_{t t}\right\|_{1}^{2}+\left\|u_{t t t}\right\|^{2}\right) d s\right)^{1 / 2}\right\}\left\|\nabla\left(\theta^{n}+\theta^{n-1}\right)\right\|,
\end{aligned}
$$

and hence

$$
\begin{aligned}
\left\|\theta^{n}\right\|_{h}^{2} \leqslant & \left\|\theta^{n-1}\right\|_{h}^{2}+C h^{4} \int_{t_{n-1}}^{t_{n}}\left\|u_{t}\right\|_{2}^{2} d s \\
& +C k^{4} \int_{t_{n-1}}^{t_{n}}\left(\left\|u_{t}\right\|_{1}^{2}+\left\|u_{t t}\right\|^{2}\right) d s,
\end{aligned}
$$

and since $\theta^{0}=0$,

$$
\left\|\theta^{n}\right\|^{2} \leqslant C h^{4} \int_{0}^{t_{n}}\left\|u_{t}\right\|_{2}^{2} d s+C k^{4} \int_{0}^{t_{n}}\left(\left\|u_{t t}\right\|_{1}^{2}+\left\|u_{t t s}\right\|^{2}\right) d s .
$$

which completes the proof of (3.12)

In order to show (3.13) it suffices, in view of (2.6) and (2.7), to deduce

$$
\begin{aligned}
\left\|\nabla \theta^{n}\right\| \leqslant & C h^{2}\left\{\left\|u_{t}(0)\right\|_{1}+\left(\int_{0}^{t_{n}}\left(\left\|u_{t}\right\|_{2}^{2}+\left\|u_{t t t}\right\|_{1}^{2}\right) d s\right)^{1 / 2}\right\} \\
& +C k^{2}\left(\int_{0}^{t_{n}}\left(\left\|u_{t t}\right\|_{2}^{2}+\left\|u_{t u t}\right\|^{2}\right) d s\right)^{1 / 2} .
\end{aligned}
$$

For this purpose, we use again (3.14), now with $\chi=\bar{\partial}_{t} \theta^{n}$, and obtain

$$
\left\|\bar{\partial}_{\imath} \theta^{n}\right\|_{h}^{2}+\frac{1}{2 k}\left(\left\|\nabla \theta^{n}\right\|^{2}-\left\|\nabla \theta^{n-1}\right\|^{2}\right)=\sum_{j=1}^{4} T_{j}^{n} .
$$


This time we estimate $T_{1}^{n}$ as

$$
\begin{aligned}
\left|T_{1}^{n}\right| & =\left|\left(\Delta\left(u^{n-1 / 2}-\left(u^{n}+u^{n-1}\right) / 2\right), \chi\right)\right| \leqslant C k \int_{t_{n-1}}^{t_{n}}\left\|u_{t t}\right\|_{2} d s\left\|\bar{\partial}_{t} \theta^{n}\right\| \\
& \leqslant C k^{3 / 2}\left(\int_{t_{n-1}}^{t_{n}}\left\|u_{t}\right\|_{2}^{2} d s\right)^{1 / 2}\left\|\bar{\partial}_{t} \theta^{n}\right\|,
\end{aligned}
$$

and obtain thus by our previous estimates for $T_{2}^{n}$ and $T_{3}^{n}$,

$$
\begin{aligned}
\left|T_{1}^{n}\right|+\left|T_{2}^{n}\right|+\left|T_{3}^{n}\right| \leqslant & C k^{3} \int_{t_{n-1}}^{t_{n}}\left(\left\|u_{t t}\right\|_{2}^{2}+\left\|u_{t t t}\right\|^{2}\right) d s \\
& +C h^{4} k^{-1} \int_{t_{n-1}}^{t_{n}}\left\|u_{t}\right\|_{2}^{2} d s+\left\|\bar{\partial} \theta^{n}\right\|_{h}^{2} .
\end{aligned}
$$

By summation we thus obtain from (3.16), since $\theta^{0}=0$,

$$
\left\|\nabla \theta^{n}\right\|^{2} \leqslant C h^{4} \int_{0}^{t_{n}}\left\|u_{t}\right\|_{2}^{2} d s+C k^{4} \int_{0}^{t_{n}}\left(\left\|u_{t t}\right\|_{2}^{2}+\left\|u_{t t}\right\|^{2}\right) d s+2 k \sum_{j=1}^{n} T_{4}^{J} .
$$

Since $T_{4}^{\prime}$ is the same as $T_{3}^{\prime}$ in the proof of Lemma 4 we now conclude from (3.8), (3.9) and (3.10),

$$
\begin{aligned}
\left\|\nabla \theta^{n}\right\|^{2} \leqslant & C h^{4}\left\{\left\|u_{t}(0)\right\|_{1}^{2}+\int_{0}^{t_{n}}\left(\left\|u_{t}\right\|_{2}^{2}+\left\|u_{t t}\right\|_{2}^{2}\right) d s\right\} \\
& +C k^{4} \int_{0}^{t_{n}}\left(\left\|u_{t}\right\|_{2}^{2}+\left\|u_{t t t}\right\|^{2}\right) d s+C k \sum_{j=1}^{n-1}\left\|\nabla \theta^{\jmath}\right\|^{2},
\end{aligned}
$$

from which (3.15) follows by the discrete Gronwall lemma. This completes the proof of the theorem.

We now turn to the explicit lumped mass forward Euler method

$$
\left(\bar{\partial}_{t} U^{n}, \chi\right)_{h}+\left(\nabla U^{n-1}, \nabla \chi\right)=\left(f^{n-1}, \chi\right) \quad \forall \chi \in S_{h} .
$$

In this case we shall need a stability condition which we state in the form

$$
\frac{k}{2}\|\nabla \chi\|^{2} \leqslant \gamma\|\chi\|_{h}^{2} \quad \forall \chi \in S_{h} \text { where } \gamma<1 .
$$

Such a condition is satisfied if $S_{h}$ satisfies the inverse estimate

$$
\|\nabla x\| \leqslant C_{0} h^{-1}\|x\|_{h},
$$

and the mesh ratio condition $k h^{-2} \leqslant 2 \gamma C_{0}^{-2}$ holds.

THEOREM 8. Let $u$ and $U^{n}$ denote the solutions of (1.1) and (3.17) with $U^{0}=R_{h} v$ and assume that the stability condition (3.18) holds. Then for $t_{n}=n k \geqslant 0$,

$$
\begin{aligned}
\left\|U^{n}-u\left(t_{n}\right)\right\| \leqslant & C h^{2}\left\{\left\|u\left(t_{n}\right)\right\|_{2}+\left(\int_{0}^{t_{n}}\left\|u_{t}\right\|_{2}^{2} d s\right)^{1 / 2}\right\} \\
& +C k\left(\int_{0}^{t_{n}}\left\|u_{t}\right\|^{2} d s\right)^{1 / 2},
\end{aligned}
$$


and if $\mathscr{T}_{h}$ is quasiuniform, for $t_{n}=n k \leqslant T$, with $C=C_{r}$,

$$
\begin{aligned}
\left\|U^{n}-u\left(t_{n}\right)\right\|_{L_{\infty}} \leqslant & C h^{2} \log \frac{1}{h}\left\|u\left(t_{n}\right)\right\|_{w_{\infty}^{2}(\Omega)} \\
& +C h^{2}\left(\log \frac{1}{h}\right)^{1 / 2}\left\{\left\|u_{t}(0)\right\|_{1}+\left(\int_{0}^{t_{n}}\left(\left\|u_{t}\right\|_{1}^{2}+\left\|u_{t}\right\|_{2}^{2}\right) d s\right)^{1 / 2}\right\} \\
& +C k\left(\log \frac{1}{h}\right)^{1 / 2}\left(\int_{0}^{t_{n}}\left\|u_{t t}\right\|^{2} d s\right)^{1 / 2} .
\end{aligned}
$$

Proof. With (3.2) valid as usual we have here

$$
\begin{aligned}
\left(\bar{\partial}_{t} \theta^{n}, \chi\right)_{h}+\left(\nabla \theta^{n-1}, \nabla \chi\right) & \left(f^{n-1}, \chi\right)-\left(\bar{\partial}_{t} R_{h} u^{n}, \chi\right)_{h}-\left(\nabla u^{n-1}, \nabla \chi\right) \\
& =\left(u_{t}^{n-1}, \chi\right)-\left(\bar{\partial}_{t} R_{h} u^{n}, \chi\right)_{h} \\
& =\left(u_{t}^{n-1}-\bar{\partial}_{t} u^{n}, \chi\right)+\left(\bar{\partial}_{t}\left(u^{n}-R_{h} u^{n}\right), \chi\right)-\varepsilon_{h}\left(\bar{\partial}_{t} R_{h} u^{n}, \chi\right) \\
& =T_{1}^{n}+T_{2}^{n}+T_{3}^{n},
\end{aligned}
$$

with the $T_{j}^{n}$ essentially as in (3.3). Setting $\chi=\theta^{n}+\theta^{n-1}$, we have

$$
\begin{gathered}
\frac{1}{k}\left(\left\|\theta^{n}\right\|_{h}^{2}-\left\|\theta^{n-1}\right\|_{h}^{2}\right)+\frac{1}{2}\left\|\nabla\left(\theta^{n}+\theta^{n-1}\right)\right\|^{2}-\frac{1}{2}\left(\left\|\nabla \theta^{n}\right\|^{2}-\left\|\nabla \theta^{n-1}\right\|^{2}\right) \\
\leqslant C\left\{h^{2} k^{-1 / 2}\left(\int_{t_{n-1}}^{t_{n}}\left\|u_{t}\right\|_{2}^{2} d s\right)^{1 / 2}\right. \\
\left.+k^{1 / 2}\left(\int_{t_{n-1}}^{t_{n}}\left\|u_{t}\right\|^{2} d s\right)^{1 / 2}\right\}\left\|\nabla\left(\theta^{n}+\theta^{n-1}\right)\right\|,
\end{gathered}
$$

whence by obvious estimates and summation, since $\theta^{0}=0$,

$$
\left\|\theta^{n}\right\|_{h}^{2} \leqslant \frac{k}{2}\left\|\nabla \theta^{n}\right\|^{2}+C h^{4} \int_{0}^{t_{n}}\left\|u_{t}\right\|_{2}^{2} d s+C k^{2} \int_{0}^{t_{n}} !_{\mid}^{\prime} u_{t t} \|^{2} d s .
$$

Using the stability assumption (3.18) now completes the proof of (3.19). Setting $\chi=\bar{\partial}_{t} \theta^{n}$ in (3.21) we have

$$
\begin{aligned}
& \left\|\bar{\partial} \theta_{t} \theta_{h}^{2}-\frac{k}{2}\right\| \nabla \bar{\partial} \dot{\theta}^{n} \|^{2}+\frac{1}{2 k}\left(\left\|\nabla \theta^{n}\right\|^{2}-\left\|\nabla \theta^{n-1}\right\|^{2}\right) \\
& \quad \leqslant C\left\{h^{2} k^{-1 / 2}\left(\int_{t_{n-1}}^{t_{n}}\left\|u_{t}\right\|_{2}^{2} d s\right)^{1 / 2}+k^{1 / 2}\left(\int_{t_{n-1}}^{t_{n}}\left\|u_{t}\right\|^{2} d s\right)^{1 / 2}\right\}\left\|\bar{\partial}_{t} \theta^{n}\right\|_{h}+T_{3}^{n} \\
& \leqslant(1-\gamma)\left\|\bar{\partial} \theta^{n}\right\|_{h}^{2}+C h^{4} k^{-1} \int_{t_{n-1}}^{t_{n}}\left\|u_{t}\right\|_{2}^{2} d s+C k \int_{t_{n-1}}^{t_{n}}\left\|u_{u}\right\|^{2} d s+T_{3}^{n} .
\end{aligned}
$$


Applying the stability condition (3.18), summing, and estimating the sum in $T 3^{j}$ as in (3.8), (3.9) and (3.10), we obtain

$$
\begin{aligned}
\left\|\nabla \theta^{n}\right\|^{2} \leqslant & C h^{4} \int_{0}^{t_{n}}\left\|u_{t}\right\|_{2}^{2} d s+C k^{2} \int_{0}^{t_{n}}\left\|u_{t t}\right\|^{2} d s+2 k \sum_{j=1}^{n} T_{3}^{J} \\
\leqslant & C h^{4}\left\{\left\|u_{t}(0)\right\|_{1}^{2}+\int_{0}^{t_{n}}\left(\left\|u_{t t}\right\|_{1}^{2}+\left\|u_{t}\right\|_{2}^{2}\right) d s\right\} \\
& +C k^{2} \int_{0}^{t_{n}}\left\|u_{t t}\right\|^{2} d s \\
& +\frac{1}{2}\left\|\nabla \theta^{n}\right\|^{2}+C k \sum_{j=1}^{n-1}\left\|\nabla \theta^{\prime}\right\|^{2} .
\end{aligned}
$$

The discrete Gronwall's lemma now yields

$$
\begin{aligned}
\left\|\nabla \theta^{n}\right\| \leqslant & C h^{2}\left\{\left\|u_{t}(0)\right\|_{1}+\left(\int_{0}^{t_{n}}\left(\left\|u_{t t}\right\|_{1}^{2}+\left\|u_{t}\right\|_{2}^{2}\right) d s\right)^{1 / 2}\right\} \\
& +C k\left(\int_{0}^{t_{n}}\left\|u_{t}\right\|^{2} d s\right)^{1 / 2},
\end{aligned}
$$

from which (3.20) follows in the standard way.

We shall now present some error estimates for our totally discrete schemes in the case of the homogeneous heat equation $(f=0$ in (1.1)) in which the regularity assumptions are somewhat weaker than so far and match those of Theorem 4 for the semidiscrete problem

$$
\begin{aligned}
\left(u_{h, t}, \chi\right)_{h}+\left(\nabla u_{h}, \nabla \chi\right) & =0 \quad \forall \chi \in S_{h}, t \geqslant 0, \\
u_{h}(0) & =v_{h} .
\end{aligned}
$$

In view of the estimates of that theorem we may restrict our attention here to the error in the discretization in time of (3.22).

We begin with the backward Euler method

$$
\begin{gathered}
\left(\bar{\partial}_{t} U^{n}, \chi\right)_{h}+\left(\nabla U^{n}, \nabla \chi\right)=0 \quad \forall \chi \in S_{h}, \\
U^{0}=v_{h},
\end{gathered}
$$

and show the following:

THEOREM 9. Let $U^{n}$ and $u_{h}$ be the solutions of (3.23) and (3.22) with $v_{h}=R_{h} v$. Then if $v=0$ on $\partial \Omega$ we have for $t_{n}=n k \geqslant 0$,

$$
\left\|U^{n}-u_{h}\left(t_{n}\right)\right\| \leqslant C k\|v\|_{2}
$$

and, if $\mathscr{T}_{h}$ is quasiuniform, for $t_{n}>0$,

$$
\left\|U^{n}-u_{h}\left(t_{n}\right)\right\|_{L_{\infty}} \leqslant C k\left(\log \frac{1}{h}\right)^{1 / 2} t_{n}^{-1 / 2}\|v\|_{2} .
$$


Proof. The proof of this result and the following will depend on spectral representations. We define a discrete Laplacian $\Delta_{h}: S_{h} \rightarrow S_{h}$ by

$$
\left(\Delta_{h} \psi, \chi\right)_{h}=-(\nabla \psi, \nabla \chi) \quad \forall \psi, \chi \in S_{h},
$$

and note that $-\Delta_{h}$ is symmetric, positive definite with respect to the inner product $(\cdot, \cdot)_{h}$ on $S_{h}$. With this notation, (3.22) may be written

$$
\bar{\partial}_{t} U^{n}=\Delta_{h} U^{n} \text {, }
$$

whence

$$
U^{n}=\left(I-k \Delta_{h}\right)^{-1} U^{n-1}=\left(I-k \Delta_{h}\right)^{-n} v_{h} .
$$

Letting now $\left\{\Lambda_{j}\right\}_{1}^{N_{h}}$ and $\left\{\Phi_{j}\right\}_{1}^{N_{h}}$ be the eigenvalues and eigenfunctions of $-\Delta_{h}$ we may write

$$
\begin{aligned}
U^{n} & =\sum_{j=1}^{N_{h}}\left(1+k \Lambda_{j}\right)^{-n}\left(v_{h}, \phi_{j}\right)_{h} \phi_{j} \\
& =-\sum_{j=1}^{N_{h}} \Lambda_{j}^{-1}\left(1+k \Lambda_{j}\right)^{-n}\left(\Delta_{h} v_{h}, \phi_{j}\right)_{h} \phi_{j},
\end{aligned}
$$

and similarly for the solution of (3.22),

$$
u_{h}\left(t_{n}\right)=-\sum_{j=1}^{N_{h}} \Lambda_{j}^{-1} e^{-n k \Lambda_{J}}\left(\Delta_{h} v_{h}, \phi_{j}\right)_{h} \phi_{j} .
$$

Hence for the error

$$
U^{n}-u_{h}\left(t_{n}\right)=-k \sum_{j=1}^{N_{h}} \delta_{n}\left(k \Lambda_{j}\right)\left(\Delta_{h} v_{h}, \phi_{j}\right)_{h} \phi_{j},
$$

where

$$
\delta_{n}(\lambda)=\lambda^{-1}\left\{(1+\lambda)^{-n}-e^{-n \lambda}\right\}
$$

We note that $\delta_{n}(\lambda)$ is bounded, uniformly in $n$, for $\lambda \geqslant 0$. In fact, for $\lambda \leqslant 1$, say, we have

$$
\begin{aligned}
\left|\delta_{n}(\lambda)\right| & =\left|\lambda^{-1}\left((1+\lambda)^{-1}-e^{-\lambda}\right) \sum_{j=0}^{n-1}(1+\lambda)^{-(n-1-j)} e^{-j \lambda}\right| \\
& \leqslant C n \lambda e^{-c n \lambda} \leqslant C,
\end{aligned}
$$

and for $\lambda>1$,

$$
\left|\delta_{n}(\lambda)\right| \leqslant 2^{-n}+e^{-n} \leqslant C .
$$

It thus follows from (3.26) that

$$
\left\|U^{n}-u_{h}\left(t_{n}\right)\right\|_{h}^{2} \leqslant C k^{2} \sum_{j=1}^{N_{h}}\left(\Delta_{h} v_{h}, \phi_{j}\right)_{h}^{2} \leqslant C k^{2}\left\|\Delta_{h} v_{h}\right\|_{h}^{2} .
$$


Here, since $\|\cdot\|$ and $\|\cdot\|_{h}$ are equivalent, we find for $v_{h}=R_{h} v$,

$$
\left(\Delta_{h} v_{h}, \chi\right)_{h}=-\left(\nabla R_{h} v, \nabla \chi\right)=-(\nabla v, \nabla \chi)=(\Delta v, \chi) \leqslant C\|\Delta v\| \cdot\|\chi\|_{h},
$$

so that

$$
\left\|\Delta_{h} v_{h}\right\|_{h} \leqslant C\|\Delta v\|,
$$

and hence altogether

$$
\left\|U^{n}-u_{h}\left(t_{n}\right)\right\| \leqslant C\left\|U^{n}-u_{h}\left(t_{n}\right)\right\|_{h} \leqslant C k\|\Delta v\|,
$$

which completes the proof of (3.24).

To show (3.25), we note that for $\chi \in S_{h}$,

$$
\|\nabla \chi\|^{2}=-\left(\Delta_{h} \chi, \chi\right)=\sum_{j=1}^{N_{h}} \Lambda_{j}\left(\chi, \phi_{J}\right)_{h}^{2} .
$$

In particular, by (3.26),

$$
\left\|\nabla\left(U^{n}-u_{h}\left(t_{n}\right)\right)\right\|^{2}=k^{2} \sum_{j=1}^{N_{h}} \Lambda_{j} \delta_{n}\left(k \Lambda_{j}\right)^{2}\left(\Delta_{h} v_{h}, \phi_{j}\right)_{h}^{2} .
$$

Now,

$$
\left|\lambda^{1 / 2} \delta_{n}(\lambda)\right| \leqslant C n^{-1 / 2} \text { for } \lambda \geqslant 0 .
$$

For, we have by (3.27), for $\lambda \leqslant 1$,

$$
\left|\lambda^{1 / 2} \delta_{n}(\lambda)\right| \leqslant C n \lambda^{3 / 2} e^{-c n \lambda} \leqslant C n^{-1 / 2},
$$

and for $\lambda>1$,

$$
\left|\lambda^{1 / 2} \delta_{n}(\lambda)\right| \leqslant \lambda^{-1 / 2}\left\{2^{-n}+e^{-n}\right\} \leqslant C n^{-1 / 2} .
$$

Hence, using also (3.28), we find for $v_{h}=R_{h} v$,

$$
\left\|\nabla\left(U^{n}-u_{h}\left(t_{n}\right)\right)\right\|^{2} \leqslant C \frac{k}{n}\left\|\Delta_{h} v_{h}\right\|_{h}^{2} \leqslant C \frac{k^{2}}{t_{n}}\|\Delta v\|^{2} \quad \text { if } v=0 \text { on } \partial \Omega .
$$

Together with (2.7) this shows (3.25) and thus completes the proof of the theorem.

We now consider the Crank-Nicolson scheme

$$
\begin{gathered}
\left(\bar{\partial}_{t} U^{n}, \chi\right)_{h}+\left(\nabla\left(U^{n}+U^{n-1}\right) / 2, \nabla \chi\right)=0 \quad \forall \chi \in S_{h}, n=1,2, \ldots, \\
U^{0}=v_{h},
\end{gathered}
$$

or with the above notation

$$
U^{n}=\left(I-\frac{1}{2} k \Delta_{h}\right)^{-1}\left(I+\frac{1}{2} k \Delta_{h}\right) U^{n-1}=r\left(-k \Delta_{h}\right)^{n} v_{h},
$$

where $r(\lambda)=\left(1+\frac{1}{2} \lambda\right)^{-1}\left(1-\frac{1}{2} \lambda\right)$. Since $|r(\lambda)|<1$ for all $\lambda>0$ this scheme is stable in $L_{2}$ and if $k \Lambda_{j}$ is bounded away from infinity it may also be expected to 
possess some smoothing property (cf. [1]). However, as max $\Lambda$, is of order $O\left(h^{-2}\right)$ this will require $k$ to be of the order of $h^{2}$ which is undesirable in applications. We shall show now that with $v \in H^{2}(\Omega)$ and $v=0$ on $\partial \Omega$ no such condition is required for the $L_{2}$ error estimate.

THEOREM 10. Let $U^{n}$ and $u_{h}$ be the solutions of (3.29) and (3.22) with $v_{h}=R_{h} v$. Then for $t_{n}=n k>0$,

$$
\left\|U^{n}-u_{h}\left(t_{n}\right)\right\| \leqslant C k^{2} t_{n}^{-1}\|v\|_{2} \text { if } v=0 \text { on } \partial \Omega .
$$

Proof. We have now

$$
U^{n}-u_{h}\left(t_{n}\right)=-k \sum_{J=1}^{N_{h}} \delta_{n}^{1}\left(k \Lambda_{\jmath}\right)\left(\Delta_{h} v_{h}, \phi_{j}\right)_{h} \phi_{j},
$$

where

$$
\delta_{n}^{1}(\lambda)=\lambda^{-1}\left\{\left(\frac{1-\frac{1}{2} \lambda}{1+\frac{1}{2} \lambda}\right)^{n}-e^{-n \lambda}\right\} .
$$

This function satisfies

$$
\left|\delta_{n}^{1}(\lambda)\right| \leqslant C n^{-1} \text { for } \lambda \geqslant 0 .
$$

In fact, for $\lambda \leqslant 1$ we have

$$
\begin{aligned}
\left|\delta_{n}^{1}(\lambda)\right| & =\left|\lambda^{-1}\left(\frac{1-\frac{1}{2} \lambda}{1+\frac{1}{2} \lambda}-e^{-\lambda}\right) \sum_{j=0}^{n-1}\left(\frac{1-\frac{1}{2} \lambda}{1+\frac{1}{2} \lambda}\right)^{n-1-j} e^{-j \lambda}\right| \\
& \leqslant C n \lambda^{2} e^{-c n \lambda} \leqslant C n^{-1}
\end{aligned}
$$

and for $\lambda \geqslant 1$,

Hence

$$
\begin{aligned}
\left|\delta_{n}^{1}(\lambda)\right| & \leqslant \lambda^{-1}\left|\left(\frac{1-2 / \lambda}{1+2 / \lambda}\right)^{n}\right|+\lambda^{-1} e^{-n \lambda} \\
& \leqslant C \lambda^{-1} e^{-c n / \lambda}+C n^{-1} \leqslant C n^{-1}
\end{aligned}
$$

$$
\begin{aligned}
\left\|U^{n}-u_{h}\left(t_{n}\right)\right\|_{h}^{2} & =k^{2} \sum_{j=1}^{N_{h}} \delta_{n}^{1}\left(k \Lambda_{j}\right)^{2}\left(\Delta_{h} v_{h}, \phi_{j}\right)_{h}^{2} \\
& \leqslant C \frac{k^{2}}{n^{2}} \sum_{j=1}^{N_{h}}\left(\Delta_{h} v_{h}, \phi_{j}\right)_{h}^{2} \\
& =C \frac{k^{4}}{t_{n}^{2}}\left\|\Delta_{h} v_{h}\right\|_{h}^{2} \leqslant C \frac{k^{4}}{t_{n}^{2}}\|\Delta v\|^{2},
\end{aligned}
$$

which completes the proof. 
In order to be able to show a similar estimate for the gradient of the error and thus also a maximum-norm error estimate we shall need to increase the smoothing power of the method. For this purpose we shall employ a device of Rannacher [5] which consists in starting the calculations by, in this case, one application of the backward Euler operator. We have then

$$
U^{n}=\left(\left(I-\frac{1}{2} k \Delta_{h}\right)^{-1}\left(I+\frac{1}{2} k \Delta_{h}\right)\right)^{n-1}\left(I-k \Delta_{h}\right)^{-1} v_{h},
$$

and we shall show the following:

THEOREM 11. Assume that the triangulation $\mathscr{F}_{h}$ is quasiuniform, let $u_{h}$ be the solution of (3.22) with $v_{h}=R_{h} v$ and let $U^{n}$ be the solution of the Crank-Nicolson scheme (3.29), modified by using in the first step the backward Euler method (3.23) and with $U^{0}=v_{h}=R_{h} v$. Then for $t_{n}=n k>0$,

$$
\left\|U^{n}-u_{h}\left(t_{n}\right)\right\|_{L_{\infty}} \leqslant C k^{2}\left(\log \frac{1}{h}\right)^{1 / 2} t_{n}^{-3 / 2}\|v\|_{2} \quad \text { if } v=0 \text { on } \partial \Omega .
$$

Proof. We have this time

$$
U^{n}-u_{h}\left(t_{n}\right)=-k \sum_{j=1}^{N_{h}} \delta_{n}^{2}\left(k \Lambda_{j}\right)\left(\Delta_{h} v_{h}, \phi_{j}\right)_{h} \phi_{j},
$$

where

$$
\delta_{n}^{2}(\lambda)=\frac{1}{\lambda}\left\{\frac{1}{1+\lambda}\left(\frac{1-\frac{1}{2} \lambda}{1+\frac{1}{2} \lambda}\right)^{n-1}-e^{-n \lambda}\right\}
$$

Here

$$
\left|\lambda^{1 / 2} \delta_{n}^{2}(\lambda)\right| \leqslant C n^{-3 / 2} \text { for } \lambda \geqslant 0
$$

since for $\lambda \leqslant 1$,

$$
\begin{aligned}
\left|\lambda^{1 / 2} \delta_{n}^{2}(\lambda)\right| & =\left|\frac{\lambda^{1 / 2}}{1+\lambda} \delta_{n-1}^{1}(\lambda)+\lambda^{1 / 2}\left(\frac{1}{1+\lambda} e^{-(n-1) \lambda}-e^{-n \lambda}\right)\right| \\
& \leqslant C n \lambda^{5 / 2} e^{-c n \lambda}+C \lambda^{3 / 2} e^{-\lambda n} \leqslant C n^{-3 / 2}
\end{aligned}
$$

and for $\lambda>1$,

$$
\left|\lambda^{1 / 2} \delta_{n}^{2}(\lambda)\right| \leqslant C \lambda^{-3 / 2} e^{-c n / \lambda}+C \lambda^{-1 / 2} e^{-n \lambda} \leqslant C n^{-3 / 2} .
$$


This yields

$$
\begin{aligned}
\left\|\nabla\left(U^{n}-u_{h}\left(t_{n}\right)\right)\right\|^{2} & =k^{2} \sum_{j=1}^{N_{h}} \Lambda_{j} \delta_{n}^{2}\left(k \Lambda_{j}\right)^{2}\left(\Delta_{h} v_{h}, \phi_{j}\right)_{h}^{2} \\
& \leqslant C \frac{k}{n^{3}} \sum_{j=1}^{N_{h}}\left(\Delta_{h} v_{h}, \phi_{j}\right)_{h}^{2} \\
& \leqslant C \frac{k^{4}}{t_{n}^{3}}\left\|\Delta_{h} v_{h}\right\|_{h}^{2} \leqslant C \frac{k^{4}}{t_{n}^{3}}\|\Delta v\|^{2},
\end{aligned}
$$

and thus shows the desired result.

We conclude by considering the forward Euler method

$$
\begin{gathered}
\left(\bar{\partial}_{l} U^{n}, \chi\right)_{h}+\left(\nabla U^{n-1}, \nabla \chi\right)=0 \quad \forall \chi \in S_{h}, \\
U^{0}=v_{h} .
\end{gathered}
$$

THEOREM 12. Let $U^{n}$ and $u_{h}$ be the solutions of (3.30) and (3.22) with $v_{h}=R_{h} v$, and assume that the stability condition (3.18) is satisfied. Then if $v=0$ on $\partial \Omega$ we have for $t_{n}=n k>0$,

$$
\left\|U^{n}-u_{h}\left(t_{n}\right)\right\| \leqslant C k\|v\|_{2},
$$

and if $\mathscr{T}_{h}$ is quasiuniform

$$
\left\|U^{n}-u_{h}\left(t_{n}\right)\right\|_{L_{\infty}} \leqslant C k\left(\log \frac{1}{h}\right)^{1 / 2} t_{n}^{-1 / 2}\|v\|_{2} .
$$

Proof. We have now

$$
U^{n}-u_{h}\left(t_{n}\right)=-k \sum_{j=1}^{N_{h}} \delta_{n}^{3}\left(k \Lambda_{j}\right)\left(\Delta_{h} v_{h}, \phi_{j}\right)_{h} \phi_{j},
$$

with

$$
\delta_{n}^{3}(\lambda)=\lambda^{-1}\left\{(1-\lambda)^{n}-e^{-n \lambda}\right\} .
$$

We note that by the stability condition (3.18),

$$
k \Lambda_{j}=-k\left(\Delta_{h} \phi_{j}, \phi_{j}\right)_{h}=k\left\|\nabla \phi_{j}\right\|^{2} \leqslant 2 \gamma .
$$

Since $\gamma<1$ we have for a sufficiently small positive $c$ that

$$
|1-\lambda| \leqslant e^{-c \lambda} \text { for } \lambda \leqslant 2 \gamma,
$$

and hence

$$
\left|\delta_{n}^{3}(\lambda)\right|=\left|\lambda^{-1}\left(1-\lambda-e^{-\lambda}\right) \sum_{j=0}^{n-1}(1-\lambda)^{n-1-\jmath} e^{-j \lambda}\right| \leqslant C n \lambda e^{-c n \lambda} \leqslant C,
$$

from which (3.31) follows as above. The proof of (3.32) is analogous. 


\section{References}

[1] G. A. Baker, J. H. Bramble and V. Thomée, "Single step Galerkin approximations for parabolic problems", Math. Comp. 31 (1977), 818-847.

[2] J. H. Bramble, A. H. Schatz, V. Thomée and L. B. Wahlbin, "Some convergence estimates for semidiscrete Galerkin type approximations for parabolic equations", SIAM J. Numer. Anal. 14 (1977), 218-241.

[3] H. Fujii, "Some remarks on finite element analysis of time-dependent field problems", in Theory and practice in finite element structural analysts (eds. Y. Yamada and R. H. Gallagher), (University of Tokyo Press, 1973), 91-106.

[4] J. H. Nitsche, " $L_{\infty}$-convergence of finite element approximations", in Mathematical aspects of finite element methods (eds. I. Galligani and E. Magenes), Lecture Notes in Math. 606 (Springer, New York, 1977), 261-274.

[5] R. Rannacher, "Discretization of the heat equation with singular initial data", Z. Angew. Math. Mech. 62 (1982), T 346-348.

[6] P. A. Raviart, "The use of numerical integration in finite element methods for solving parabolic equations", in Topics in numerical analysis (ed. J. J. H. Miller), (Academic Press, 1973), 233-264.

[7] M. Tabata, " $L^{\infty}$-analysis of the finite element method", in Lecture notes in numerical and applied analysis 1 (eds. H. Fujita and M. Yamaguti), (1979), 25-62.

[8] P. Tong, T. H. H. Pian and L. L. Bucciarelli, "Mode shapes and frequencies by finite element method using consistent and lumped masses", Comput. \& Structures 1 (1970), 623-638.

[9] T. Ushijima, "On the uniform convergence for the lumped mass approximation of the heat equation", J. Fac. Sci. Univ. Tokyo 24 (1977), 477-490.

[10] T. Ushijima, "Error estimates for the lumped mass approximation of the heat equation", Mem. Numer. Math. 6 (1979), 65-82.

[11] M. F. Wheeler, “A priori $L_{2}$ error estimates for Galerkin approximations to parabolic partial differential equations", SIAM J. Numer. Anal. 10 (1973), 723-759.

[12] M. F. Wheeler, " $L_{\infty}$ estimates of optimal order for Galerkin methods for one dimensional second order parabolic and hyperbolic equations", SIAM J. Numer, Anal. 10 (1973), 908-913. 